\title{
VLTI monitoring of the dust formation event of the Nova V1280 Scorpii ${ }^{\star}$
}

\author{
O. Chesneau ${ }^{1}$, D. P. K. Banerjee ${ }^{2}$, F. Millour ${ }^{3}$, N. Nardetto ${ }^{3}$, S. Sacuto ${ }^{1}$, A. Spang ${ }^{1}$, M. Wittkowski ${ }^{4}$, N. M. Ashok ${ }^{2}$,
} R. K. Das ${ }^{2}$, C. Hummel ${ }^{4}$, S. Kraus ${ }^{3}$, E. Lagadec ${ }^{5}$, S. Morel ${ }^{6}$, M. Petr-Gotzens ${ }^{4}$, F. Rantakyro ${ }^{6}$, and M. Schöller ${ }^{6}$

1 UMR 6525 H. Fizeau, Univ. Nice Sophia Antipolis, CNRS, Observatoire de la Côte d'Azur, Av. Copernic, 06130 Grasse, France e-mail: Olivier.Chesneau@obs-azur.fr

2 Physical Research Laboratory, Navrangpura, Ahmedabad, Gujarat, India

3 Max-Planck-Institut für Radioastronomie, Auf dem Hügel 69, 53121 Bonn, Germany

${ }^{4}$ European Southern Observatory, Karl-Schwarzschild-Strasse 2, 85748 Garching bei München, Germany

5 Jodrell Bank Center for Astrophysics, The University of Manchester, Oxford Street, Manchester M13 9PL, UK

${ }^{6}$ European Southern Observatory, Casilla 19001, Santiago 19, Chile

Received 30 January 2008 / Accepted 25 April 2008

\section{ABSTRACT}

\begin{abstract}
Context. We present the first high spatial-resolution monitoring of the dust-forming nova V1280 Sco, performed with the Very Large Telescope Interferometer (VLTI).

Aims. These observations promise to improve the distance determination of such events and constrain the mechanisms leading to very efficient dust formation under the harsh physical conditions encountered in novae ejecta.

Methods. Spectra and visibilities were regularly acquired between the onset of dust formation, 23 days after discovery (or 11 days after maximum), and day 145, using the beam-combiner instruments AMBER (near-IR) and MIDI (mid-IR). These interferometric observations were complemented by near-infrared data from the $1.2 \mathrm{~m}$ Mt. Abu Infrared Observatory, India. The observations are initially interpreted in terms of simple uniform models; however more complex models, probably involving a second shell, are required to explain data acquired following $t=110 \mathrm{~d}$ after outburst. This behavior is in accordance with the light curve of V1280 Sco, which exhibits a secondary peak at about $t=106 \mathrm{~d}$, followed by a new, steep decline, suggesting a new dust-forming event. Spherical dust shell models generated with the DUSTY code are used to investigate the parameters of the main dust shell.

Results. Using uniform disk models, these observations allow us to determine an apparent linear expansion rate for the dust shell of $0.35 \pm 0.03$ mas day $^{-1}$ and the approximate ejection time of the matter in which dust formed of $t_{\text {ejec }}=10.5 \pm 7 \mathrm{~d}$, i.e. close to the maximum brightness. This information, combined with the expansion velocity of $500 \pm 100 \mathrm{~km} \mathrm{~s}^{-1}$, implies a distance estimate of $1.6 \pm 0.4 \mathrm{kpc}$. The sparse $u v$ coverage does not enable deviations from spherical symmetry to be clearly discerned. The dust envelope parameters were determined. The dust mass generated was typically $2-8 \times 10^{-9} M_{\odot}$ day $^{-1}$, with a probable peak in production at about 20 days after the detection of dust and another peak shortly after $t=110 \mathrm{~d}$, when the amount of dust in the shell was estimated as $2.2 \times 10^{-7} M_{\odot}$. Considering that the dust-forming event lasted at least 200-250 d, the mass of the ejected material is likely to have exceeded $10^{-4} M_{\odot}$. The conditions for the formation of multiple shells of dust are also discussed.
\end{abstract}

Key words. techniques: interferometric - techniques: high angular resolution - stars: individual: V1280 Sco stars: novae, cataclysmic variables - stars: circumstellar matter - stars: mass-loss

\section{Introduction}

Infrared observations of classical novae have established that dust grains can condense in the harsh environment formed in the ejecta generated by thermonuclear runaways on the surfaces of white dwarfs in close binary systems (Gehrz 1988; Gehrz et al. 1998). The formation and evolution of dust grains, following a nova outburst, remains a difficult problem both from the observational and the theoretical point of view. The typical light curve during dust formation shows a deep minimum lasting for months; the observed timescale for dust formation is short - typically 1-20 days - indicating an efficient process of dust formation. Most dust-forming novae condense amorphous carbon, which produces a featureless gray body or black-body

* Based on observations made with the Very Large Telescope Interferometer at Paranal Observatory under programs 278.D-5053, 279.D-5014 and 079.D-0415. spectral energy distribution (SED). These appear to result from thermonuclear runaways on low-mass, carbon-oxygen $(\mathrm{CO})$ WDs that tend to eject more mass under less energetic conditions than novae on oxygen-neon-magnesium (ONeMg) WDs (Schwarz et al. 1997, 2001, 2007; Rudy et al. 2003). V705 Cas (Nova Cas 1993) is probably the most studied case showing such a phenomenon (Gehrz et al. 1995; Evans et al. 1996, 2005).

The application of optical interferometers to the study of nova eruptions, provides not only information on the diameter and shape of the ejecta (Quirrenbach et al. 1993; Lane et al. $2007 \mathrm{~b}$ ), but also on the physical processes at work. The stateof-the-art facility of the VLTI, both in terms of infrastructure and management of the observations, allows one to schedule rapidly evolving novae observations (Schöller et al. 2006). A snapshot of the outburst early phase $(t=5.5 \mathrm{~d})$ of the famous recurrent nova RS Oph was captured by AMBER, the near-IR VLTI recombiner; this observations revealed the complex geometry and kinematics of the ejection at a spectral resolution 
of 1500 (Chesneau et al. 2007). Monnier et al. (2006) and Lane et al. (2007a) described the monitoring of the RS Oph outburst using several near-IR optical interferometers during two months following the 2006 outburst. These observations allowed them to follow the expansion of the source and estimate the free-free emission from the nova wind. RS Oph was also observed in the $N$ band with the Keck Interferometer Nuller $\sim 3.8$ days after discovery; these observations provided evidence that some nebular lines and hot dust were present outside the obscured area, i.e. before the arrival of the blast wave of the outburst (Barry et al. 2008).

V1280 Sco was discovered in outburst by Yamaoka et al. (2007) on 2007 February 04.86, about 12 days before reaching its maximum in visual light $\left(m_{\mathrm{V}} \sim 4\right)$. This climb to maximum was exceptionally slow, and the nova also exhibited an unusual characteristic by forming dust as soon as two weeks after maximum. The first description of its infrared spectrum can be found in Rudy et al. (2007). Das et al. (2007) described near-infrared JHK spectroscopy of V1280 Sco, obtained with the Mt. Abu 1.2-m telescope, which displayed several strong C I emission lines. Further spectra, obtained on 2007 March 04.95, showed a sharply-rising continuum in the $J, H$, and $K$ bands, indicating dust formation in the nova ejecta, a few days after distinct change was observed in the slope of the visual light curve.

We report on the first infrared interferometry observations ever recorded of a dust-forming nova, providing a unique set of spectra and visibilities obtained during the first 145 days of the eruption of V1280 Sco. It was initially planned to use exclusively the VLTI/AMBER near-IR combiner; when the nova continued to produce dust at a high rate, most of the observations were acquired instead using the VLTI/MIDI mid-IR combiner. We followed the expansion of the dust shell for more than one hundred days and detected the appearance of a new shell. This allows us to constrain simultaneously the extension and content of the newly-formed dust by means of simple geometric models and also by using the public-domain DUSTY code (Ivezic et al. 1999; Ivezic \& Elitzur 1997). We emphasize however, that the parameter space characterizing the dust shells was not extensively covered and that the parameters proposed in the present article must be considered as indicative only, pending further detailed investigations. The outline of the paper is as follows: in Sect. 2, we present the analysis of the interferometric and spectroscopic data, in Sect. 3, we present a semi-quantitative interpretation of the event by means of the light curve and associated classical relations, in Sect. 4, we analyze the interferometric data using simple geometrical models, and in Sect. 5, an attempt is made to investigate further the shell parameters using the DUSTY code. Finally, we discuss in Sect. 6 some mechanisms for dust formation around novae that are able to account for the observed multiple shell behavior; our conclusions are presented in Sect. 7 .

\section{Observations}

\subsection{A complex light curve}

Once a nova has been discovered, its light curve provided mostly by amateur observations, is the first measurement used to infer the nature of the event. The light curve of V1280 Sco is shown in Fig. 1. This curve was extracted from the AFOEV database, operated at $\mathrm{CDS}^{1}$. The first notable feature is the slow rise to visual maximum lasting about 12 days since the discovery was

\footnotetext{
${ }^{1}$ http://cdsweb.u-strasbg.fr/cgi-bin/afoevList?sco/ v1280
}

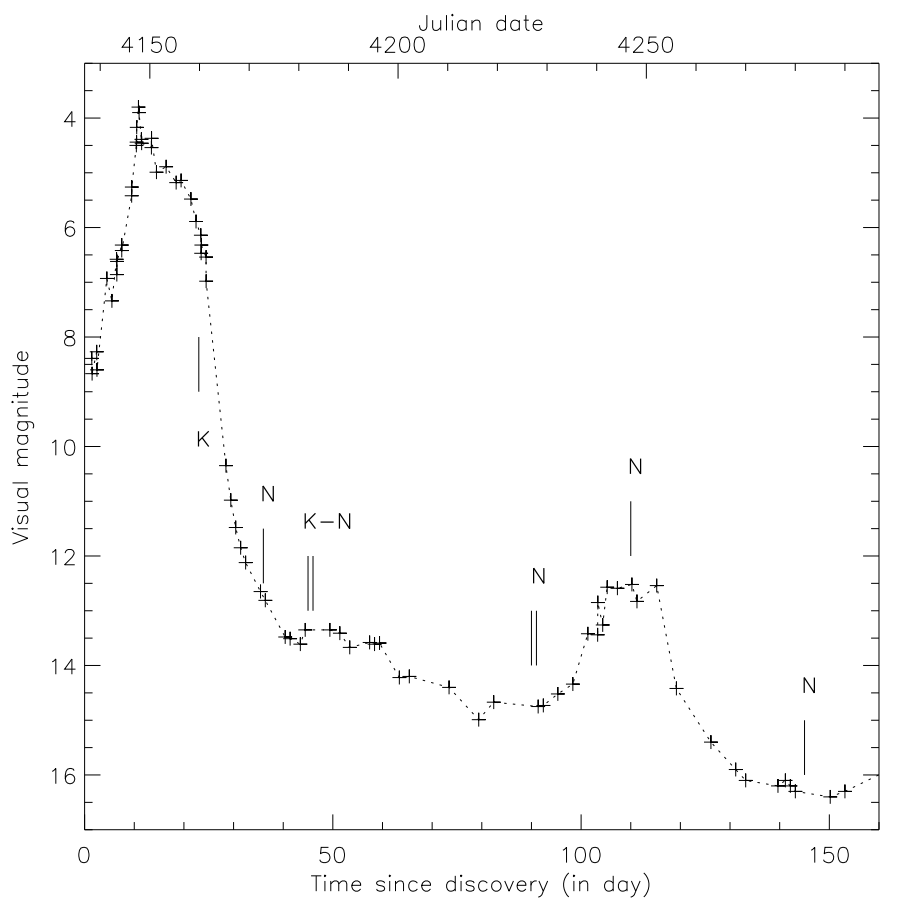

Fig. 1. The visual light curve of V1280 Sco from AFOEV data with the dates of the present VLTI observations superposed on it. The time for the light curve to drop by 2 mag from maximum, $t_{2}$, seems to occur after the beginning of dust formation which is suggested by the sharp change in slope of the light curve.

reported. Very few novae exhibited such long premaximum behavior, which is found generally only in slow novae. The maximum of visual light on 16 February 2007 is preceded by a sharp rise in brightness, from the 5 th mag to the maximum magnitude $m_{\mathrm{v}} \sim 3.8$. This is followed by a smoother decline lasting less than 10 days, which was interrupted abruptly by the dust formation event. The source faded over the next few days from the 7 th to the 12 th mag, then continued to fade at a lower rate to reach and stabilize at magnitude 15 between $t=80$ and $t=90$ days (2007-04-27/2007-05-06). A second maximum occurred, peaking close to $t=104 \mathrm{~d}$ (2007-05-20) with a magnitude $m_{\mathrm{V}}=12.5$, followed again by a sharp decline to 16 th mag. The sequence of our observations was as follows. The first AMBER observations were performed on 28 February 2007, when the nova had not formed dust yet. The next set of observations, originally planned with AMBER, were completed on 13 March with MIDI because it appeared that dust was forming at a high rate. The first MIDI observations were successfully performed with the $1.8 \mathrm{~m}$ Auxiliary Telescopes (ATs). These observations were difficult to reduce because the $N$ band flux of V1280 Sco of 15-20 Jy was close to the MIDI sensitivity limit. Only the visibility measurement performed at the lower airmass $(\sim 1.1)$ could be used scientifically, the two other measurements had error bars that were too large. On 22 March, AMBER observations with 2 ATs were performed followed by MIDI observations on 23 March. In May, the visual flux dropped below the sensitivity limit of $m_{\mathrm{V}}=13.5$, which is required to acquire the source on the ATs. Therefore, the observations were then conducted using the $8.2 \mathrm{~m}$ Unit Telescopes (UTs), although the source at this epoch was even brighter in the $N$ band, rising to $m_{N}=-1.5$ on 26 May. We were fortunate to acquire observations during some important moments in the evolution of the nova. The first $K$ band observations were completed at $t=23 \mathrm{~d}$ (2007-02-28) just before the dust formation event, while the subsequent observations 
Table 1. Observing log reporting the interferometric observations used in the paper. In the column UTC Date are reported the date and hour of observation in the format year-month-day-T-hour. On the right are reported the different telescopes and stations used: the $8.2 \mathrm{~m}$ fixed unit telescopes are labeled with $\mathrm{U}$ and the number of the telescope; the stations of the movable auxiliary telescopes are labeled with the letters $\mathrm{A}$, G, $\mathrm{H}$ and $\mathrm{K}$ and a number. The reader can have an overview of the array in the following link: http://www. eso.org/observing/etc/doc/vlti/ baseline/vltisations.html

\begin{tabular}{|c|c|c|c|c|c|c|c|c|c|}
\hline \multirow[t]{2}{*}{ Julian day } & \multirow[t]{2}{*}{2007 UTC Date } & \multirow[t]{2}{*}{ Day $^{1}$} & \multirow[t]{2}{*}{ Instrument } & \multirow[t]{2}{*}{ Magnitude } & \multirow{2}{*}{$\begin{array}{c}\text { Telescope } \\
\text { stations }\end{array}$} & \multicolumn{2}{|c|}{ Projected baseline } & \multirow[t]{2}{*}{ Airmass } & \multirow{2}{*}{$\begin{array}{c}\text { Closest } \\
\text { Calibrator }^{2}\end{array}$} \\
\hline & & & & & & $\begin{array}{l}\text { Length } \\
\text { [meter] }\end{array}$ & $\begin{array}{c}\text { PA } \\
\text { [degrees] }\end{array}$ & & \\
\hline 2454160.4 & $2007-02-28 \mathrm{~T} 09$ & 23 & AMBER (K) & $3.8(K)$ & G1-H0 & 71 & 175 & 1.14 & HD 151680 \\
\hline 2454173.3 & 2007-03-13Т08 & 36 & MIDI (N) & $1(N)$ & G0-K0 & 60.2 & 53 & 1.10 & HD 150798 \\
\hline 2454181.3 & 2007-03-22Т07 & 45 & $\operatorname{AMBER}(\mathrm{K})$ & $4.2(K)$ & G0-H0 & 29.0 & 46 & 1.21 & HD 149447 \\
\hline 2454182.4 & 2007-03-23T08 & 46 & $\operatorname{MIDI}(\mathrm{N})$ & $0.3(N)$ & A0-G0 & 62.7 & 61 & 1.04 & HD 150798 \\
\hline 2454182.4 & 2007-03-23T09 & 46 & MIDI (N) & $0.3(N)$ & A0-G0 & 63.9 & 67 & 1.01 & HD 150798 \\
\hline 2454226.7 & 2007-05-06T05 & 90 & MIDI (N) & $-0.8(N)$ & U3-U4 & 60.1 & 102 & 1.04 & HD 163376 \\
\hline 2454226.9 & 2007-05-06T09 & 90 & MIDI (N) & $-0.8(N)$ & U3-U4 & 57.9 & 132 & 1.18 & HD 163376 \\
\hline 2454227.8 & 2007-05-07T08 & 91 & MIDI (N) & $-0.8(N)$ & U3-U4 & 59.3 & 128 & 1.11 & HD 163376 \\
\hline 2454246.5 & 2007-05-26T01 & 110 & MIDI (N) & $-1.5(N)$ & U3-U4 & 35.0 & 75 & 1.69 & HD 163376 \\
\hline 2454281.5 & 2007-06-30T00 & 145 & MIDI (N) & $-1.1(N)$ & U3-U4 & 52.8 & 93 & 1.17 & HD 123139 \\
\hline 2454281.7 & 2007-06-30T05 & 145 & MIDI (N) & $-1.1(N)$ & U3-U4 & 58.7 & 129 & 1.15 & HD 163376 \\
\hline 2454281.8 & 2007-06-30T07 & 145 & MIDI (N) & $-1.1(N)$ & U3-U4 & 49.5 & 166 & 2.07 & HD 177716 \\
\hline
\end{tabular}

${ }^{1}$ From discovery, Feb. 4.85 UT. JD = 2454136.85

${ }^{2}$ HD 151680 (K2.5III, $5.83 \pm 0.06$ mas), HD150798 (K2III, $8.76 \pm 0.12$ mas), HD149447 (K6III, $4.55 \pm 0.05$ mas); HD 163376 (M0III, $3.79 \pm$ 0.12 mas), HD123139 (K0III, $5.12 \pm 0.02$ mas), HD177716 (K1III, $3.72 \pm 0.07$ mas).

cover the evolution from $t=36 \mathrm{~d}(2007-03-13)$ to the minimum light at $t=90 \mathrm{~d}$ (2007-05-06). One observation was recorded close to the top of the second maximum at $t=110 \mathrm{~d}(2007-05-$ 26), while the last set of observations at $t=145 \mathrm{~d}(2007-06-30)$ cover the second deep minimum.

The sequence of VLTI observations is described in Table 1. We used the VLT Unit Telescopes (8.2 m, UTs), whose stations are labeled U2, U3 or U4 in the table and the Auxiliary Telescopes (1.8 m, ATs), which were located at stations A0, G0, G1, H0, K0. The spectrally-dispersed visibilities are shown in Fig. 2.

\section{2. $A M B E R$ data}

The AMBER data were recorded using the combination of 2 telescopes only, since the three-telescope mode was difficult to organize and operate under conditions with a sense of urgency needing observations to be planned at short notice ${ }^{2}$. For similar reasons, the data were secured using the lowest spectral resolution $(\lambda / \delta \lambda=35)$. The AMBER data were reduced using the amdlib software developed by the AMBER consortium, following the scheme described by Tatulli et al. (2007). The squaredvisibility estimator was computed from the basic observables provided by the adopted algorithm: the coherent flux (i.e. complex visibilities obtained frame by frame multiplied by the flux) and the estimated fluxes from each telescope.

These low-resolution data were reduced by applying the "standard" AMBER data reduction recipes: files were selected when the optical-path difference measurement did not exceed half the coherence length $\left(\lambda^{2} / \delta \lambda\right)$; frame selection was then applied, in which $80 \%$ of the 'bad frames were rejected, according to the fringe-contrast SNR measurement explained in Millour et al. (2007). Only one calibration star was observed for each night, HD 151680 (K3III, $5.93 \pm 0.06$ mas) and HD 149447 (K6III, $4.55 \pm 0.05$ mas), at $t=23 \mathrm{~d}$ and $t=45 \mathrm{~d}$, respectively, and accounting for the error bars and systematics was not

\footnotetext{
${ }^{2}$ For instance, the need to accurately set the Optical Path Delay model for the new baselines using several observations of the calibrators.
}

straightforward. We considered a conservative value of $10 \%$ for the relative accuracy of $V^{2}$, which was much larger than calculated from internal dispersion of the squared visibilities (4\% for the first dataset and $2.5 \%$ for the second). On other nights, the transfer function scatter is of the order of $10 \%$ rms (i.e. 0.1 for a transfer function of 1 and 0.05 for a transfer function of 0.5 ).

The calibrated visibility of V1280 Sco on February 28 is very close to 1 , meaning that the object is unresolved within the large error-bar estimates. The same comment applies to the night of 2007 March 22, but as the calibration star is smaller and the visibility of V1280 Sco is also smaller, the corresponding error was less prominent.

We attempted to determine if evidence of a rising dust continuum could be found in the first $K$-band AMBER data by completing a (crude) flux calibration using interferometric calibrators as flux standards. The 28 Feb. spectrum shows clusters of emission lines, which are not resolved in the low spectralresolution mode, and a decreasing continuum towards longer wavelengths. The spectrum taken 45 days after discovery is far smoother and a significant increase in flux toward longer wavelengths is observed.

\subsection{MIDI data}

The VLTI/MIDI interferometer operates in a similar way to a classical Michelson interferometer by combining the mid-IR light ( $N$ band, 7.5-13.5 $\mu \mathrm{m}$ ) from two UTs or two ATs. We used a typical MIDI observing sequence, as described in Ratzka et al. (2007), which is briefly summarized here. Chopped acquisition images were recorded $(f=2 \mathrm{~Hz}, 2000$ frames, $4 \mathrm{~ms}$ per frame, 98 mas per pixel) for the fine acquisition of the target. The beam combiner and dispersion unit were then introduced into the optical path and the interference pattern was scanned by means of a temporal modulation of the path by a reflective device mounted on a piezo motor. The lower spectral resolution provided by a prism was used mostly, which corresponded to a spectral dispersion $\lambda / \delta \lambda$ of about 30 ; some data however were recorded using the high spectral mode provided by a grism $(\lambda / \delta \lambda=$ 230) although we note that all data recorded were spectrally 


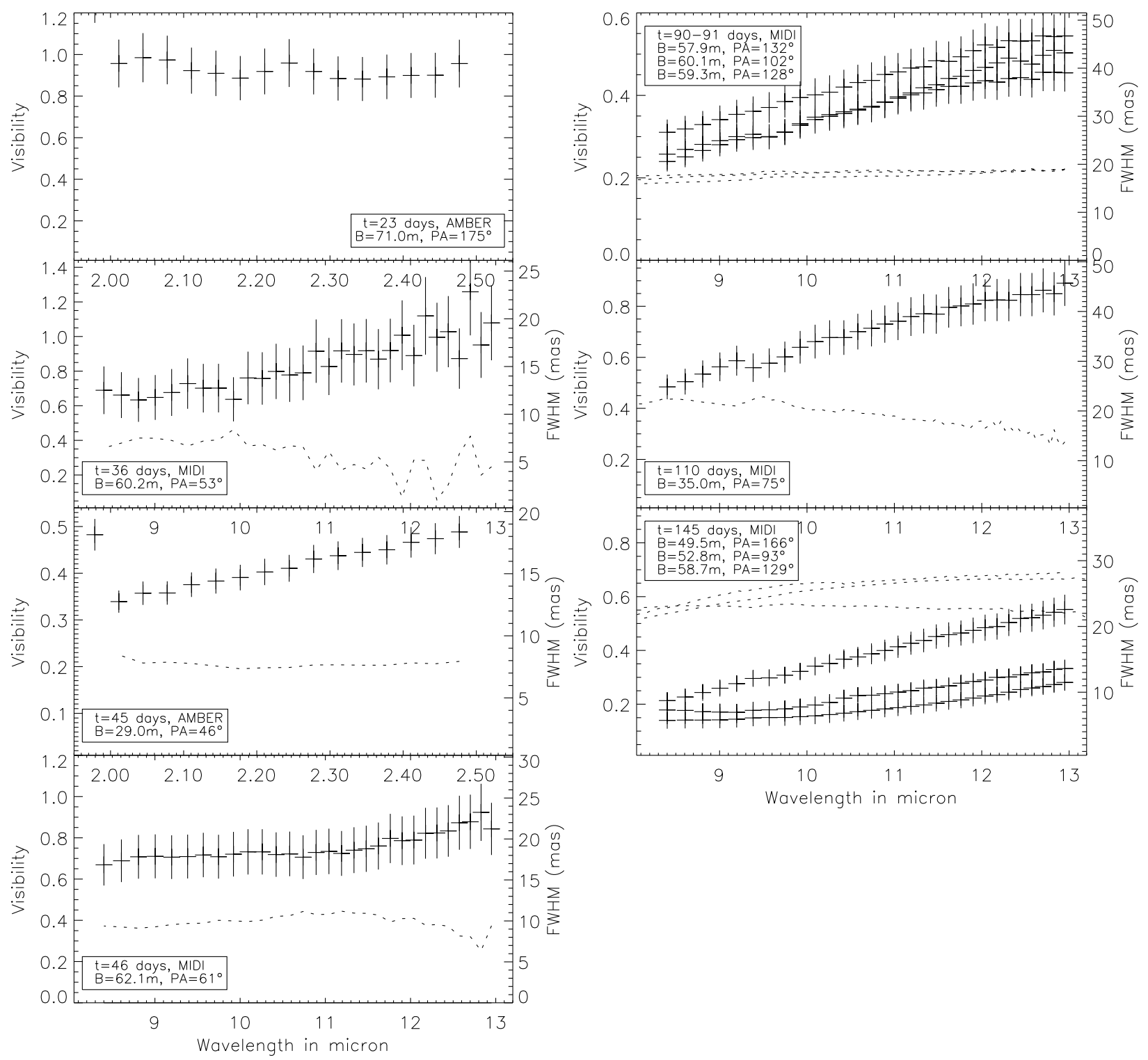

Fig. 2. Full data set of visibilities recorded with MIDI and AMBER, from the earliest (top, left) to the latest dates (bottom, right). The visibilities are indicated by the crosses (left vertical axis), and estimates of the size of the source, using Gaussian models is shown in dotted lines (right vertical axis).

featureless. The data-reduction software packages $^{3}$ MIA and EWS were used to reduce the spectra and visibilities.

Most of the MIDI observations of V1280 Sco were performed in the so-called HIGH_SENS mode, which implies that the photometry of each telescope was recorded subsequently to the fringe acquisition. The errors, including the internal and calibrator diameter uncertainties, range from $20 \%$ for the first dataset, when the source $N$ band flux was at the limit of sensitivity of AT telescopes; then $10-15 \%$ for the AT observations and about $7 \%-15 \%$ for the UT observations. The accuracy of the absolute flux calibration is $10-15 \%$, but the measurements of the spectra local slopes are accurate at the percent level. The fluxes are shown in Fig. 3, with the fits to the data using black-body curves for an estimate of their apparent temperature.

Accurate differential phase curves could be extracted with EWS from the high quality data recorded on the 6th of May and later. The curves from V1280 Sco are smooth and dominated by

\footnotetext{
${ }^{3}$ http://wWw.mpia-hd.mpg.de/MIDISOFT/, http://WwW. strw.leidenuniv.nl/\$ sim\$nevec/MIDI/
}

atmospheric residuals of the same order as that arising from the calibrators. The corrected differential phase is typically $0^{\circ} \pm 5$, for each day of observation. These measurements indicate that the source image does not significantly depart from point symmetry, but does not provide any information on possible departures from spherical geometry (e.g. bipolar geometry). The EWS software removes a large part of the low frequency phase signal, but a strong phase signal from dusty disks can still be detected unambiguously (Deroo et al. 2007).

\subsection{Near-Infrared spectroscopy}

Since its outburst in February 2007, near-infrared JHK observations of V1280 Sco were made on a regular basis from the $1.2 \mathrm{~m}$ telescope at the Mt. Abu Observatory, India. A full description of these results is completed elsewhere (Das et al. 2008) although an early set of spectra, taken soon after the outburst, is described by Das et al. (2007a,b). In the present work, we show in Fig. 4, representative $J$ band spectra of V1280 Sco 


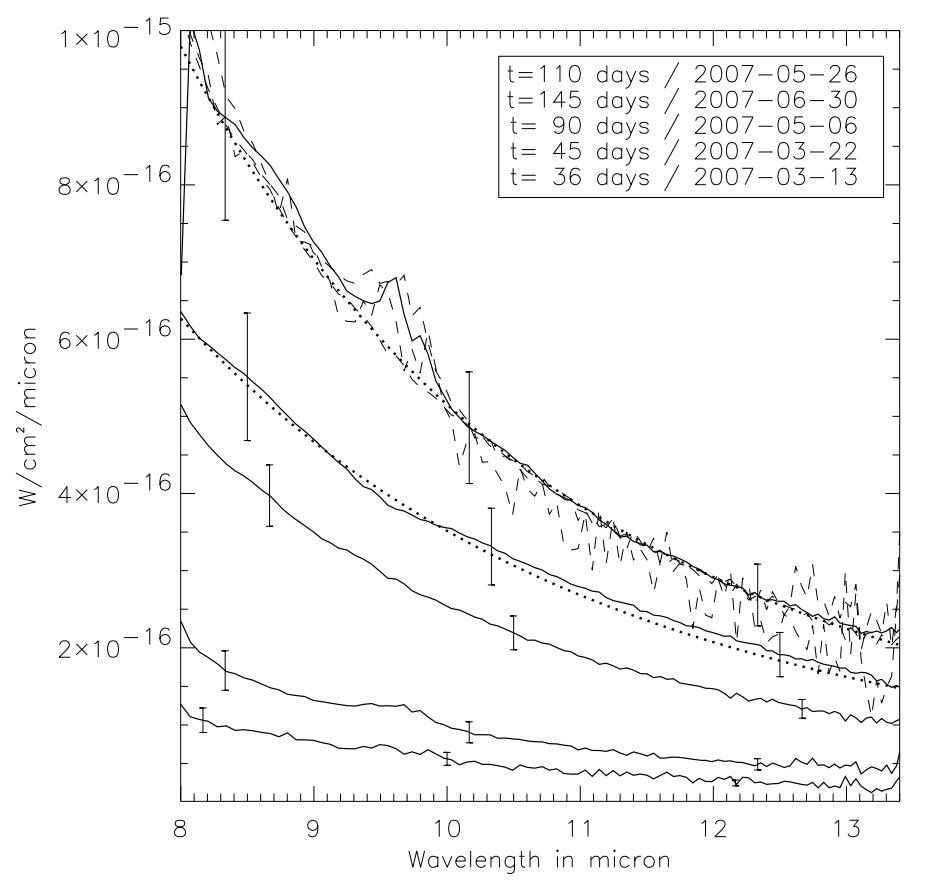

Fig. 3. From bottom to top, flux calibrated mid-IR spectra recorded on days $36,45,90,145$ and 110 . The dashed lines superimposed on the maximum flux recorded at day 110 are the fluxes at $t=36,45$ and 90 days after scaling. The slopes of the four spectra are similar within error bars indicating an invariant temperature for the dust continuum. The $t=110$ days curve is well fitted by a black-body with $T=920 \mathrm{~K}$ while that for $t=145$ days exhibits a slightly different slope which is well fitted by a black-body with $T=760 \mathrm{~K}$. The two fits are indicated in dotted lines.

taken on 26 February 2007 and 7 March 2007. The observations were obtained with the Near Infrared Imager/Spectrometer with a $256 \times 256 \mathrm{HgCdTe}$ NICMOS3 array at a resolution of approximately $10 \AA /$ pixel. The $J$ band spectra are typical of a classical nova soon after outburst, showing prominent lines of HI, HeI, OI and CI. The presence of strong neutral CI lines is invoked subsequently (while modeling the dust properties) to be indicative that carbon is the likely principal constituent of the dust that formed around V1280 Sco. The significant change seen in the slope of the continuum, between the two epochs of observations in Fig. 4, is also indicative of the formation of dust around this time (this is also manifested by a sharp decline in the optical light curve at approximately this epoch). The later spectrum of 7 March shows a steepening of the continuum towards longer wavelengths i.e. the building up of an infrared excess which is expected during the onset of dust formation. Several $K$ band spectra, recorded between February 18 and June 8 2007, are shown in Das et al. (2008). The February 27 spectrum exhibits a strong Br $\gamma$ lines peaking at twice the continuum level, and few weaker broad lines. The Br $\gamma$ line reached a maximum on March 1, peaking at 3.4 above the continuum and then decreases markedly. The spectrum recorded on March 13 is almost featureless, dominated by the dust continuum. The first AMBER data were secured the 28 February, before the onset of dust formation. The second, acquired March 13, probes the dusty shell.

\section{Semi-quantitative interpretation}

The interferometric observables in the infrared domain provide a fresh insight into the dust shells formed around the novae that complements information derived from the flux-calibrated

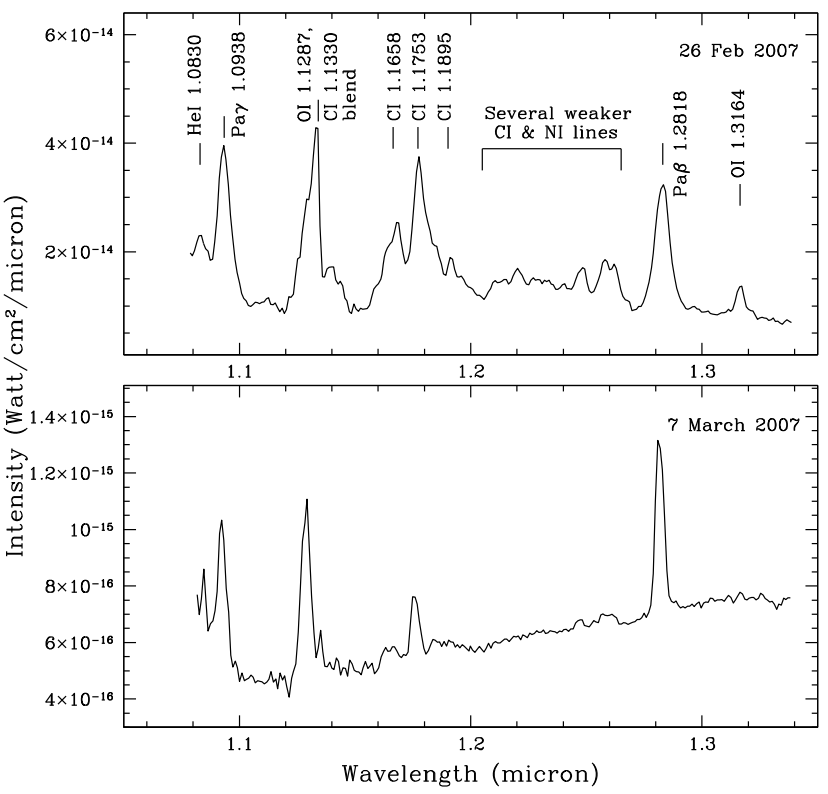

Fig. 4. $J$ band spectrum of V1280 Sco on February $26.97(t=22 \mathrm{~d}$, top panel) and March 7.95, 2007 ( $t=38 \mathrm{~d}$, bottom panel $)$ are shown. The prominent emission lines are marked. The formation of dust between the two epochs is evidenced clearly from the rising of continuum towards longer wavelengths in the later spectrum of 7 March 07, while no dust signature is visible in the first spectrum.

spectra. The modeling of the dust shell involves many parameters, representing the variable temperature and brightness of the source, the nature of the dust, and the density law of the dustforming region. The visibilities per se do not however allow the degeneracies in the observable to be resolved. As a consequence, it becomes necessary to estimate a few parameters using classical relations and velocity estimations to use them as a first guess in the fitting process.

\subsection{First estimation of the basic parameters: classical relations}

Munari et al. (2007a) measured a $B$ magnitude of 4.1 and a $V$ magnitude of 3.79 for V1280 Sco at the time of maximum visual light (the 16th of Feb.). A few days before maximum, the temperature was close to that of a F-type star (Yamaoka et al. 2007); a temperature of $5300 \mathrm{~K}$ was also estimated by Rudy et al. (2007). We assume that the temperature at maximum was about $T_{0}=7000 \mathrm{~K}$. Assuming a $B-V \sim 0 / 0.3$ (for an A or F spectral type), we estimate $E(B-V)$ to be 0.3 or slightly less, and $A_{V}$ to be $0.9-1.1$. As a cross-check, the Galactic extinction law from Marshall et al. (2006), provides a $K$ absorption coefficient $A_{K}=0.107$ to 0.127 between 1 and $8 \mathrm{kpc}$, scaling to $A_{V}=1.2-1.4$ (assuming $A_{V} / A_{K}=11$ ).

We use the relationship between absolute magnitude and rate of decline (the so-called MMRD relations, della Valle \& Livio 1995) to obtain an estimate of the absolute magnitude and the distance of the source. The estimation of the $t_{2}$ and $t_{3}$ values that is the time that the nova required to fade by 2 and by 3 mag, respectively, becomes complicated because of the sudden drop in the light curves, which interrupted the regular fading trend, caused by the fast formation of dust. If we ignore dust formation and extrapolate the light curve to determine when 2 mag of fading occurs, then 21 days appears as an appropriate 
Table 2. Results from the conversion of the MIDI visibilities by means of geometric models. The quoted errors are at the $1 \sigma$ level. The lines indicated by an asterisk, close to the data, present the extension of the model wherein we assume an additional unresolved source representing $10 \%$ of the total flux at $t=110 \mathrm{~d}$ and $15 \%$ at $t=145 \mathrm{~d}$ (keeping in mind that the total flux from the unresolved source is a lower estimate of the true flux of the appearing compact source). For further details please see the text.

\begin{tabular}{|c|c|c|c|c|c|c|c|c|c|c|c|}
\hline \multirow[t]{2}{*}{$\overline{\overline{\text { Day }}}$} & \multirow[t]{2}{*}{ 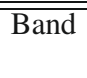 } & \multicolumn{2}{|c|}{ Projected baseline } & \multicolumn{4}{|c|}{ Uniform disk diameter (mas) } & \multicolumn{4}{|c|}{$\overline{\text { Gaussian } F W H M \text { (mas) }}$} \\
\hline & & $B(\mathrm{~m})$ & $\mathrm{PA}\left(\left(^{\circ}\right)\right.$ & $2.2 \mu \mathrm{m}$ & $8 \mu \mathrm{m}$ & $10 \mu \mathrm{m}$ & $13 \mu \mathrm{m}$ & $2.2 \mu \mathrm{m}$ & $8 \mu \mathrm{m}$ & $10 \mu \mathrm{m}$ & $13 \mu \mathrm{m}$ \\
\hline 23 & $\bar{K}$ & 71 & 175 & $\leq 1.5$ & & & & $\leq 1.0$ & & & \\
\hline 36 & $N$ & 60.2 & 53 & & $8 \pm 3.0$ & $10 \pm 3.0$ & $7 \pm 4.0$ & & $5 \pm 2.0$ & $7 \pm 2.0$ & $5 \pm 3.0$ \\
\hline 45 & $K$ & 29 & 46 & $12.5 \pm 2$ & & & & $7.5 \pm 1$ & & & \\
\hline 46 & $N$ & 62.7 & 61 & & $13 \pm 1.5$ & $13 \pm 1.5$ & $12 \pm 2.5$ & & $8 \pm 4.0$ & $8 \pm 4.0$ & $7 \pm 4.0$ \\
\hline 90 & $N$ & 60.1 & 102 & & $25 \pm 0.5$ & $28 \pm 0.5$ & $30 \pm 1.0$ & & $17 \pm 1.0$ & $18 \pm 1.0$ & $19 \pm 1.0$ \\
\hline 90 & $N$ & 57.9 & 132 & & $27 \pm 1.0$ & $28 \pm 1.0$ & $30 \pm 1.0$ & & $18 \pm 2.0$ & $19 \pm 2.5$ & $19 \pm 3$ \\
\hline 91 & $N$ & 59.3 & 128 & & $26 \pm 1.5$ & $30 \pm 1.5$ & $32 \pm 2.0$ & & $18 \pm 1.5$ & $19 \pm 1.5$ & $21 \pm 2$ \\
\hline 110 & $N$ & 35.0 & 75 & & $35 \pm 3.0$ & $34 \pm 3.0$ & $32 \pm 3.0$ & & $22 \pm 2.0$ & $20 \pm 2.0$ & $15 \pm 2.5$ \\
\hline $110^{*}$ & $\mathrm{~N}$ & 35.0 & 75 & & $43 \pm 3.0$ & $43 \pm 3.0$ & $42 \pm 3.0$ & & $27 \pm 2.0$ & $27 \pm 2.0$ & $27 \pm 2.0$ \\
\hline 145 & $N$ & 52.8 & 93 & & $37 \pm 1.0$ & $44 \pm 1.0$ & $47 \pm 2.0$ & & $22 \pm 1.5$ & $26 \pm 1.5$ & $28 \pm 1.0$ \\
\hline $145^{*}$ & $N$ & 52.8 & 93 & & - & $48 \pm 1.0$ & $51 \pm 2.0$ & & $\geq 35$ & $33 \pm 1.5$ & $33 \pm 1.0$ \\
\hline 145 & $N$ & 58.7 & 129 & & $36 \pm 1.5$ & $40 \pm 1.5$ & $45 \pm 2.0$ & & $21 \pm 1.0$ & $24 \pm 1.0$ & $27 \pm 1.5$ \\
\hline $145^{*}$ & $N$ & 58.7 & 129 & & - & - & $50 \pm 2.0$ & & $\geq 35$ & $33 \pm 1.5$ & $34 \pm 1.0$ \\
\hline 145 & $N$ & 49.5 & 166 & & $38 \pm 4.0$ & $39 \pm 3.0$ & $37 \pm 3.0$ & & $23 \pm 1.0$ & $23 \pm 1.0$ & $22 \pm 1.5$ \\
\hline $145^{*}$ & $\mathrm{~N}$ & 49.5 & 166 & & $42 \pm 1.0$ & $42 \pm 2.0$ & $42 \pm 2.0$ & & $30 \pm 1$ & $27 \pm 1.0$ & $26 \pm 1.5$ \\
\hline
\end{tabular}

estimate. For $A_{V}=1.2$, the MMRD relations yield $D=1.3 \mathrm{kpc}$ for $t_{2}=21$ days and $D=1.7 \mathrm{kpc}$ for $t_{2}=13$ days, which is the shortest estimate allowed by the dust-forming event. Two rates of decline can be extracted: one applicable from $t=11 \mathrm{~d}$ (maximum light) to $t \sim 16 \mathrm{~d}$, with a rapid decline of $0.24 \mathrm{mag} / \mathrm{day}$, and a far slower rate from this point to $t \sim 21 \mathrm{~d}$, of less than $0.1 \mathrm{mag} / \mathrm{day}$. The curve trend is then changed abruptly by the dust-forming event.

In Naito \& Narusawa (2007), the FWHM of the $\mathrm{H} \alpha$ emission on Feb. 5.87 UT was $400 \mathrm{~km} \mathrm{~s}^{-1}$, and its absorption minimum is blueshifted by $480 \mathrm{~km} \mathrm{~s}^{-1}$ from the emission peak. Low-resolution spectra acquired on Feb. 12.88 and 14.83 UT (Yamaoka et al. 2007) show Balmer lines with clear P-Cyg profiles and an expansion velocity of about $500 \mathrm{~km} \mathrm{~s}^{-1}$. Munari et al. (2007a) reported that, on an average, the displacement of the P-Cyg absorptions from the emission components was $595 \mathrm{~km} \mathrm{~s}^{-1}$. The Br $\gamma$ line also showed during the initial days a marked P Cygni profile centered at about $575 \mathrm{~km} \mathrm{~s}^{-1}$ (Das et al. 2008). Thus, for the estimation of the size of the ejecta, we will adopt a conservative velocity value of $500 \pm 100 \mathrm{~km} \mathrm{~s}^{-1}$.

\section{Fitting the interferometric data with geometric models}

We interpret the visibility curves using simple geometric models, assuming a uniformly-distributed or Gaussian-like radiallysymmetric flux distribution. Table 2 displays the results of the fitting at selected wavelengths.

\subsection{The dust formation event}

Before the dust formation event, the $K$ band continuum was expected to be dominated by free-free emission from the nova wind (Gehrz 1988). We obtained a single $K$ band visibility curve with AMBER at $t=23 \mathrm{~d}$ using two telescopes that tightly constrained the pseudo-photosphere size before or near the dust formation event. The $K$ band visibility was $0.96 \pm 0.12$ and the projected baseline was $71 \mathrm{~m}$. This implies that this is an unresolved, compact source, given the large error bar at this date, that has a typical diameter smaller than approximately 1 mas. This upper limit translates into a linear size of 1-2 AU for a corresponding distance value of 1-2 kpc.

As can be seen in Fig. 4, the AMBER spectrum does not show any rising continuum at $t=23 \mathrm{~d}$, in line with the Mt. Abu spectroscopic observations recorded two days earlier, although a small contribution from an optically-thin, extended dust shell in formation cannot be excluded. We note that the limited spectral resolution does not allow us to isolate emission lines that are still observable at that stage of the outburst (see Fig. 4), albeit with a weak impact on the observables (contribution of a few percent).

The dust forms rapidly an optically-thick dust shell whose effects are detectable from the visible to the $N$ band; a uniform disk provides a good approximation for the source light distribution (see Fig. 5). The MIDI visibilities on day 36 were inaccurate, whereas on day 46 they were more tightly constrained, in close agreement with AMBER observations. On day 90, the MIDI spectrally dispersed visibilities appeared to be described more accurately by a Gaussian model than a uniform disk, although it is difficult to compare these models, given the accuracy of the measurements.

On $t=110$ day, a difficulty arises in interpreting the $35 \mathrm{~m}$ baseline visibility curve, which exhibits a slope that departs significantly from a uniform disk or Gaussian model. In an attempt to solve this issue, we introduce a new unresolved component representing about $10-15 \%$ of the flux. The residual of the curve is well fitted with a Gaussian of FWHM 27 mas or a uniform disk of 43 mas. This hypothesis is validated in view of the three visibility curves secured at $t=145 \mathrm{~d}$ (see Fig. 6) with baselines ranging from $49 \mathrm{~m}$ to $59 \mathrm{~m}$. At this date, the shell should have been as extended as $\sim 50$ mas, and with these baselines, the shell should have been almost fully resolved. One can see that the three curves are not at the low level expected and converge to a level of $V \sim 0.15$ at $8 \mu \mathrm{m}$. We infer that another compact source, of $F W H M=13-17$ mas, contributes $\sim 10-20 \%$ of the $N$ band flux, by calculating the level of the curve and the slight differences between the visibilities of the different baselines (which are nevertheless lower than the error-bar uncertainty). An alternative interpretation of the $t=110 \mathrm{~d}$ single visibility curve would be possible, but the presence of a new dust shell is established beyond any doubt from the $t=145 \mathrm{~d}$ data. This compact component is probably the dust forming region, as its size 
coincides with the size of the first detection of the shell at $t=23 \mathrm{~d}$, that can be seen as the extended ejecta become optically thin in the $N$ band.

\subsection{The shell expansion and distance determination}

From the angular diameters provided in Table 2, we infer the expansion rate of the main shell by interpolating the points using a linear expansion curve. This can be done at several chosen wavelengths viz. at 8, 10 and $13 \mu \mathrm{m}$; each of these wavelengths is affected by slightly different errors and bias. The first observations at $t=36 \mathrm{~d}$ were very noisy and the observations at $t=110 \mathrm{~d}$ and $t=145 \mathrm{~d}$ suffer from contamination introduced by the presence of the compact inner regions that have to be carefully taken into account. This leads to the large uncertainty in the curves. We tested three methods for determining the linear fit of the expansion rate based on three sets of data. The first one considered only the data points taken before $t=110 \mathrm{~d}$ to avoid the points affected by the appearance of the second shell. The second set includes all visibility measurements, assuming simple uniform disk models, while the third data set uses the diameters of the main shell corrected for the presence of a compact source (indicated with an asterisk in Table 2). The curves based on the second set indicate that the time of ejection is significantly before the discovery of the outburst: this is unrealistic and shows that the data recorded at $t=110 \mathrm{~d}$ and $t=145 \mathrm{~d}$ should be corrected for the presence of a compact additional component. At $13 \mu \mathrm{m}$, the curves based on the first set of data, and on the full corrected dataset agree fairly well, while at $8 \mu \mathrm{m}$, there are significant discrepancies. This difficulty is related to the large errors in the first measurement at $t=36 \mathrm{~d}$.

The first conclusion is that the probable time of ejection is closer to the maximum of brightness than the date of discovery, with a computed mean date of $t_{\text {ejec }}=10.5 \pm 7 \mathrm{~d}$ for the zero crossing of the expansion curve. The mean expansion rate is determined with an accuracy better than $10 \%$ at $0.35 \pm 0.03$ mas day $^{-1}$. As often reported, the main source of error in the distance determination comes from estimates of the dust shell velocity. The distance inferred from the expansion, assuming a velocity of the dust shell of $500 \pm 100 \mathrm{~km} \mathrm{~s}^{-1}$ is $1.6 \pm 0.4 \mathrm{kpc}$. This value roughly agrees with estimates based on the MMRD relations.

\subsection{Testing the spherical symmetry hypothesis}

The resolved remnants of novae ejecta provide evidence that nova shells often depart from spherical symmetry. This may point either to the fact that the eruption is intrinsically not spherically symmetric or that these large-scale observations probe the interaction of the shell with its nearby interstellar environment. The spatially-resolved observations of the recurrent nova RS Oph showed that the ejection was highly asymmetric, rapidly forming a nebula characterized by two prominent lobes and a dense equatorial waist (Bode et al. 2007; Chesneau et al. 2007; O'Brien et al. 2006). This spatial complexity was visible from the very beginning of the outburst (Chesneau et al. 2007).

The sparse VLTI observations provide little information about the shape of the source. Three telescopes could not be used simultaneously, preventing closure phase spectra from being recorded. None of the 12 MIDI differential phases departs significantly from zero, with an error bar lower than $5^{\circ}$. This eliminates some geometries such as a binary signal with moderate flux ratio, but does not provide any constraint on the shape of a centrally-symmetric flux source. The last set of visibilities recorded at $t=145 \mathrm{~d}$ provide an indication of departure from sphericity that appears significant with respect to the error bars. Three baselines were used, covering about $75^{\circ}$ with similar projected lengths. The baselines at $\mathrm{PA}=93^{\circ}$ and $129^{\circ}$ could be fitted by the same double-shell model, while another set of parameters, involving a smaller shell is necessary to explain the level of the baseline at PA $=166^{\circ}$. However, a strong offset of this visibility curve is possible considering that this point was recorded at the end of the night with an airmass of 2 and in HIGH_SENS mode (i.e. with separated photometry), while the other two set of observations were performed using the SCI_PHOT mode (i.e. with simultaneous photometry).

In conclusion, the hypothesis that the ejection might have been aspherical is not constrained by the VLTI data and in absence of further information a spherical geometry is considered. As a consequence, it is not possible to apply the corrections proposed in Wade et al. (2000) for taking into account the asphericity of the source in the computation of the distance.

\section{Fitting the data with DUSTY models}

The use of uniform or Gaussian disk models should be justified by a more physical model of the expanding dust shell. Uniform disks are probably the most appropriate models to describe the optically-thick dust shell, while the Gaussian distribution may be more appropriate to describe the later stages of the shell evolution, when it dilutes and becomes optically thin. We attempt to infer the parameters of the dust shell by using a spherical radiative-transfer code.

DUSTY is a public-domain simulation code that models radiation transport in a circumstellar dusty environment by analytically integrating the radiative-transfer equation in plane-parallel or spherical geometries (Ivezic et al. 1999; Ivezic \& Elitzur 1997). The code utilizes the self-similarity and scaling relation of the radiatively-heated dust, such that the shell is efficiently characterized by its optical depth. This means that absolute values (e.g. luminosity and dust-shell dimensions) are not uniquely determined by the transfer problem and must be inferred by external constraints - in our case, the recorded MIDI visibilities and flux-calibrated spectra. The synthetic spectrally-dispersed visibility profiles throughout the $N$ band $(7.5-13 \mu \mathrm{m})$ are generated using a set of 20 wavelengths and compared with the MIDI visibilities for each baseline. At $t=45-46 \mathrm{~d}$, the $2.2 \mu \mathrm{m} K$ band wavelength is also included to account for the AMBER measurement.

The following assumptions are made for the numerical simulations with DUSTY:

- spherical symmetry. This point is weakly constrained by our observations as discussed in Sect. 4.3;

- the dust shell is homogeneous (i.e. not clumpy);

- the source is point-like as viewed from the shell. This assumption is probably inaccurate, since the central source, in the first moments of the outburst, is a pseudo-photosphere surrounded by a screen of the close-by ejected material, in which molecules and dust can form. This assumption is even more restrictive when considering the effect of a complex density structure (i.e. multiple shells) in the emerging SED and visibilities (see Discussion),

- the temperature and the spectrum of the source is constrained by model fitting. First simple black-body fits were used in the models, but free-free curves provided much higher quality fits (see following section); 
- the dust is made of amorphous carbon. There is no other realistic possibility in the absence of dust features in the MIDI spectra. The near-IR spectra exhibited some carbon lines indicative of the presence of a CO nova (Das et al. 2007), which disappeared rapidly to be replaced by a featureless continuum;

- the grain size distribution follows the classical MRN (Mathis, Rumpl \& Nordsieck 1977) relation: $n(a) \propto$ $a^{-q}$ for $a_{\min } \leq a \leq a_{\max }$. Only $q$ was considered as a free parameter during the fitting process, while the values $a_{\text {min }}=0.03 \mu \mathrm{m}$ and $a_{\max }=3 \mu \mathrm{m}$ were kept fixed;

- the thickness of the dust shell $\left(R_{\text {out }} / R_{\text {in }}\right)$ is constrained mainly by the visibilities. Inside this shell, the density follows a $r^{-2}$ distribution as in a steady-state wind with constant velocity. We performed many tests and the effects of changing the power of the density are difficult to evaluate: the effect is small for a geometrically thin shell and is relatively larger in the later stages of evolution.

\subsection{Simulating a single shell}

The parameters that provided satisfactory fits to the data are shown in Table 2. The most complete set of observations was obtained at $t=45-46 \mathrm{~d}$ for which we have, in addition to the $J H K$ photometry, both MIDI and AMBER observations, which provide similar estimates of the shell size in $K$ and $N$ bands. This is a strong indication that the dust shell was optically thick and geometrically thin at that time.

The information extracted from this limited exercise can be summarized as follows:

- the central source SED must depart strongly from that of a black-body. Unrealistically low temperatures were often required to achieve good fits using black-body spectra, in line with comments reported for the modeling of other novae using similar SEDs (Harrison et al. 1998) ${ }^{4}$. The assumption that the central source radiation is converted into free-free emission appears a good alternative because the inner zones of the ejecta absorb completely the flux from the hot WD and emit subsequently free-free emission (Gehrz 1988). Indeed, free-free spectra improved significantly the quality of the fits. The temperature associated with the free-free spectra ranged between 7500 to $10000 \mathrm{~K}$. This corresponded to temperatures associated with regions that are ionized by hot central sources, such as HII regions, planetary nebulae and Be star disks in which the gas temperature is usually close to $10000 \mathrm{~K}$ (see Banerjee et al. 2001). Similar quality fits are obtained when changing from a low-temperature source SED to a higher one if one increases the optical depth, such that the source temperature cannot be measured reliably;

- No grain size distributions with values of $q$ above 3 were able to provide good fits to the SEDs ${ }^{5}$. This implies that the size of grains distribution is weighted towards large grains, from the start of observations. We find values of $q \sim 2.1$ from the earliest dates that agree well with the range found in Evans et al. (2005), but slightly larger values around $q \sim 3$ seem necessary at later time. This suggests that processes decreasing the size of the dust grains were operating just after their

\footnotetext{
${ }^{4}$ Note that in this paper the authors tried to improve the fits by using two black-body curves at $9000 \mathrm{~K}$ and $3000 \mathrm{~K}$ in an attempt to split the source between the outburst core and its compact vicinity.

5 We do not consider the last model proposed for $t=145 \mathrm{~d}$ as a viable solution.
}

formation. This behavior is opposite to that reported in Evans et al. (1997, Fig. 8);

- the very first moments of dust-shell formation were captured by the VLTI interferometer as dust continuously formed. As a consequence, the thickness of the shell (i.e. $Y=1+\Delta r / r$ ) was constantly increasing. However, we note that the SED fitting is not much affected by this parameter $Y$; some family of solutions being found both for narrow (as low as $\sim 1.05$ ) as for wide values of $Y$. However, the observed visibilities constrain tightly the values reported in Table 3 . These large shell thicknesses are different from the narrow ones encountered for the modeling of a dust shell observed a long time after the outburst, whose value is set by the duration of the dust-forming event and the internal velocity dispersion of the ejecta;

- it was impossible to find solutions for all dates for which the luminosity of the central source was unchanged. This is related to several factors: the temperature and SED of the central source are not constrained well at these stages; the luminosity in the DUSTY code determines both the flux level and the position of the shell inner radius. Therefore, the luminosity is an essential ingredient for the fitting of both spectra and visibilities. The difficulties encountered with this parameter illustrate the limits of the model (see Sect. 5.4);

- as expected, single-shell models have the highest difficulties in accounting for the last visibilities recorded at $t=145 \mathrm{~d}$ although, some models are able to provide visibilities that mimic the plateau observed at the 0.15 level. We have to extend the shell thickness to unrealistically high values of $Y$ which correspond to the outer radii reaching 30-40 mas. This model probably bears some resemblance with reality in the sense that the inner radius and its vicinity accounts for the new shell, while the outer parts of the shell provides the screening of the first shell.

We also note that the spectral shape of the MIDI visibilities, recorded at $t=36 \mathrm{~d}$ and $t=46 \mathrm{~d}$, are not smooth whereas the visibilities later exhibit a behavior more in line with the models. The obvious explanation is that these measurements are noisy because the source was close to the sensitivity limit of the $1.8 \mathrm{~m}$ Auxiliary Telescopes. This argument is surely valid for the high level visibilities recorded from $10 \mu \mathrm{m}$ to $13 \mu \mathrm{m}$. Nevertheless, the quality of the data is slightly better in the short wavelength range, and the visibilities recorded at $t=36 \mathrm{~d}$ are lower than expected by the model or by the trend of the expansion curves shown in Fig. 5. A physical interpretation can be tentatively proposed, in the form of an additional opacity from molecular bands of various species, that contributes to screen the central source differently depending on the wavelength, although the molecules responsible from such a signal inside carbon rich ejecta are not well defined. It is not possible to state precisely whether this signal is effectively seen here, but this potentiality must be investigated in the future. We note also that the smoothness of the high SNR visibility curves, obtained after $t=90 \mathrm{~d}$, provide an upper limit to the clumpiness of the shell although this limit excludes only the presence of a few, bright, mas-scale dust structures in the shell.

\subsection{Complex density profiles}

From day 110 on, the simple single-shell models are no longer able to account for the shape of the visibility curves, while being still able to fit the SEDs. Changing the power of the density profile for the full shell does not bring any significant improvement, 


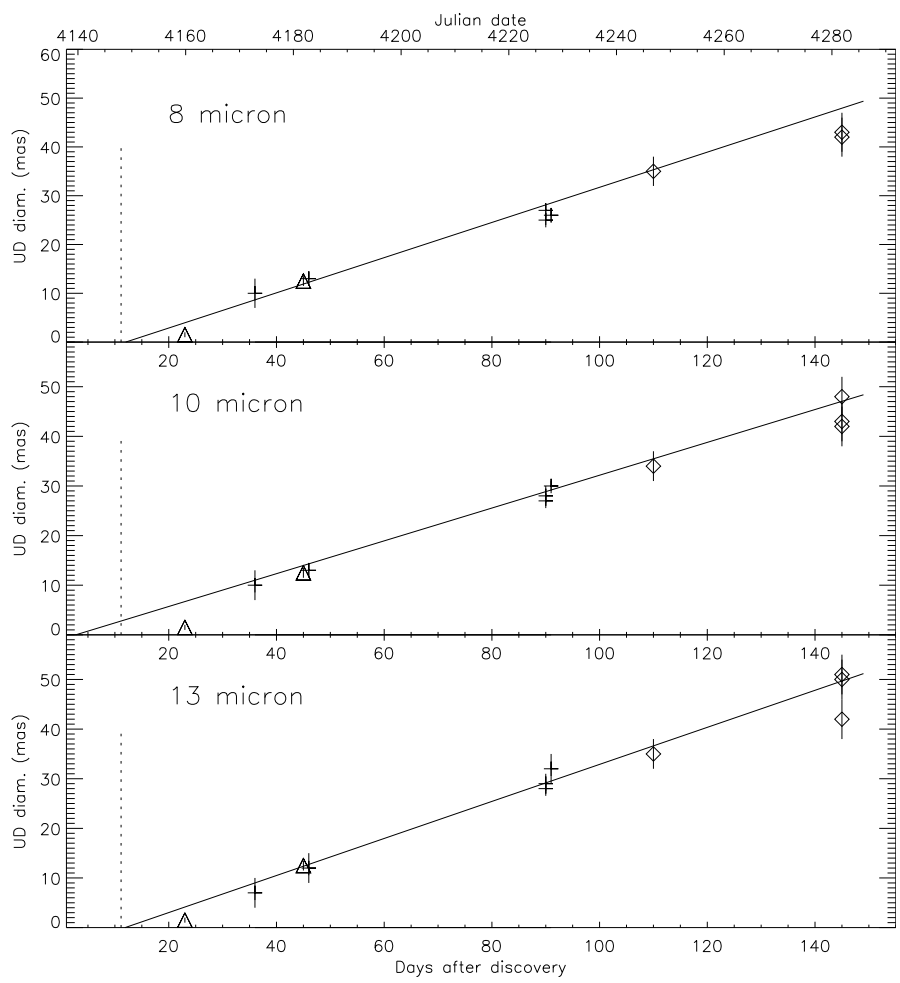

Fig. 5. Uniform disk (UD) diameters at $8 \mu \mathrm{m}$ (top), $10 \mu \mathrm{m}$ and $13 \mu \mathrm{m}$ (bottom) as a function of time, together with the best polynomial fits of a linear expansion model. The time of maximum light $(t=11 \mathrm{~d})$ is shown as a dotted vertical line. The points are directly inferred from the visibilities using simple UD models (crosses), while the squares, representing the diameters measured after $t=110 \mathrm{~d}$ are corrected from the presence of a compact, unresolved source. Taking this correction into account (and its potentially large associated error), one can see that the first dust shell originates from material launched a few days before the maximum light. The AMBER measurements at $t=23 \mathrm{~d}$ and $t=$ $45 \mathrm{~d}$ are shown as triangles (these points are not included in the fitting process).

although we suspect that the solution may reside in a complex density law that would reflect the variations in the rate of dust formation with time. As shown previously, there is some evidence in the latest visibilities recorded that some $N$ band emission originates in more compact regions than the extended shell and we tried to model this by inserting a second shell in the DUSTY code. We first attempted to use a tabulated profile with two discrete shells but this kind of density profiles is not suited for the code. We also tried to assume a monotonic $r^{-2}$ density profile with density enhancements of the kind used in Blöcker et al. (2001). However, the monotonic density profile dominated the visibilities and the number of new parameters was large. We finally tried a third approach, still far from being self-consistent. The radiative transfer in the second (young) shell was first computed, and a SED and the visibilities were stored. The parameters of this shell were poorly constrained. The outcoming flux was then injected into the parameters in a second simulation in which the first extended shell was included, although we stress that DUSTY took into account only the shape of the injected SED. There is no way to constrain the parameters of this shell, but we note that the parameters of the $t=36 \mathrm{~d}$ shell might represent a good approximation given the estimations of the compact-source size. The visibilities generated were biased: this was because the extension of the inner shell was not taken into account by the code in the second simulation, and the flux contribution of the first shell had also to be carefully taken into account for computing the flux ratio between the two shells. The last option, used for the $t=145 \mathrm{~d}$ models, was to test different density profiles from $r^{-3}$ to $r^{-1}$, and broken power-laws. Families of satisfactory models can be found and we propose one of them in the last column of Table 3. This example is an extreme case: the velocity implied by the extension of the outer radius is about twice the rate inferred from the uniform disk models and the temperature is very high. Moreover, the value of $q$ is most probably overestimated because the SED fit is obviously less satisfactory. Nevertheless, it is worth noting that the mass inferred for this shell is in close agreement with the first option. At some point in the evolution of the dust-forming nova envelope, it is certain that the Center to Limb Variation (CLV) has evolved in a complex manner not accounted for by the simple models presented in this paper. Defining physically a "second" shell-forming event might be an over-interpretation of the data. Given the amount of open issues and the number of free parameters involved, the possibilities are almost infinite, while the data remains fairly limited. We did not try to investigate this point further - it deserves an extensive study which is beyond the scope of the paper.

\subsection{Mass of the shell}

The DUSTY models provide a good estimate of the shell mass and hence the dust formation rate during the event. The mass in the circumstellar dust shell was computed for each of the models following the relation for a $r^{-2}$ power-law dust density distribution (Sarkar \& Sahai 2006):

$M_{\mathrm{dust}}=4 \pi R_{\mathrm{in}}^{2} Y\left(\tau_{100} / \kappa_{100}\right)$

where $R_{\text {in }}$ is the inner radius of the dust shell (see Table 3), $Y$ is the relative shell thickness $(1+\Delta R / R), \tau_{100}$ is the shell optical depth at $100 \mu \mathrm{m}$ and $\kappa_{100}$ is the dust mass absorption coefficient at $100 \mu \mathrm{m}$. The value of $\kappa_{100}$ depends on the size of the grains, which varies in our models. We have used the values computed for amorphous carbon, which range between $58 \mathrm{~cm}^{2} \mathrm{~g}^{-1}$ for $q=$ 2.1 (mean size $0.006 \mu \mathrm{m}$ ) and $66 \mathrm{~cm}^{2} \mathrm{~g}^{-1}$ for $q=3$ (mean size $0.003 \mu \mathrm{m})$.

We estimate, with large errors, the amount of dust formed per day in between each observation. From the first shell modeled, we measured $3.1 \times 10^{-9} M_{\odot}$ day $^{-1}$, while between $t=36 \mathrm{~d}$ and $t=45 \mathrm{~d}$, it was found to reach $7.4 \times 10^{-9} M_{\odot}$ day $^{-1}$. Between $t=45 \mathrm{~d}$ and $t=110 \mathrm{~d}$, the dust formation rate was $1.8 \times 10^{-9} M_{\odot}$ day $^{-1}$. This suggest that the dust-forming process may have slowed down in that time interval, in line with the null forming rate estimated between $t=90 \mathrm{~d}$ and $t=110 \mathrm{~d}$. Given the uncertainties in the data, the modeling, and the date when dust began to form, these estimates must be interpreted with some caution. The lower amount of mass found at $t=145 \mathrm{~d}$ is also less significant than the other estimates due to the difficulty in finding visibilities that match the spectral shape of the observations. Using a modeling that has two distinct shells increases the amount of dust predicted considerably.

It is probable that dust formation continued for several weeks before the rebrightening of the source in October-November 2007 (Munari et al. 2007b). If we assume a mean value of dustforming rate of $5 \times 10^{-9} M_{\odot}$ day $^{-1}$, and a canonical gas-to-dust ratio of 150 (although this number can be much larger, see Gehrz (1988)) then a dust shell of $1 \times 10^{-4} M_{\odot}$ is generated in about 130 days. V1280 Sco could most probably have ejected a mass of material that exceeds this number during the 200-250 days of the dust shell presence. 

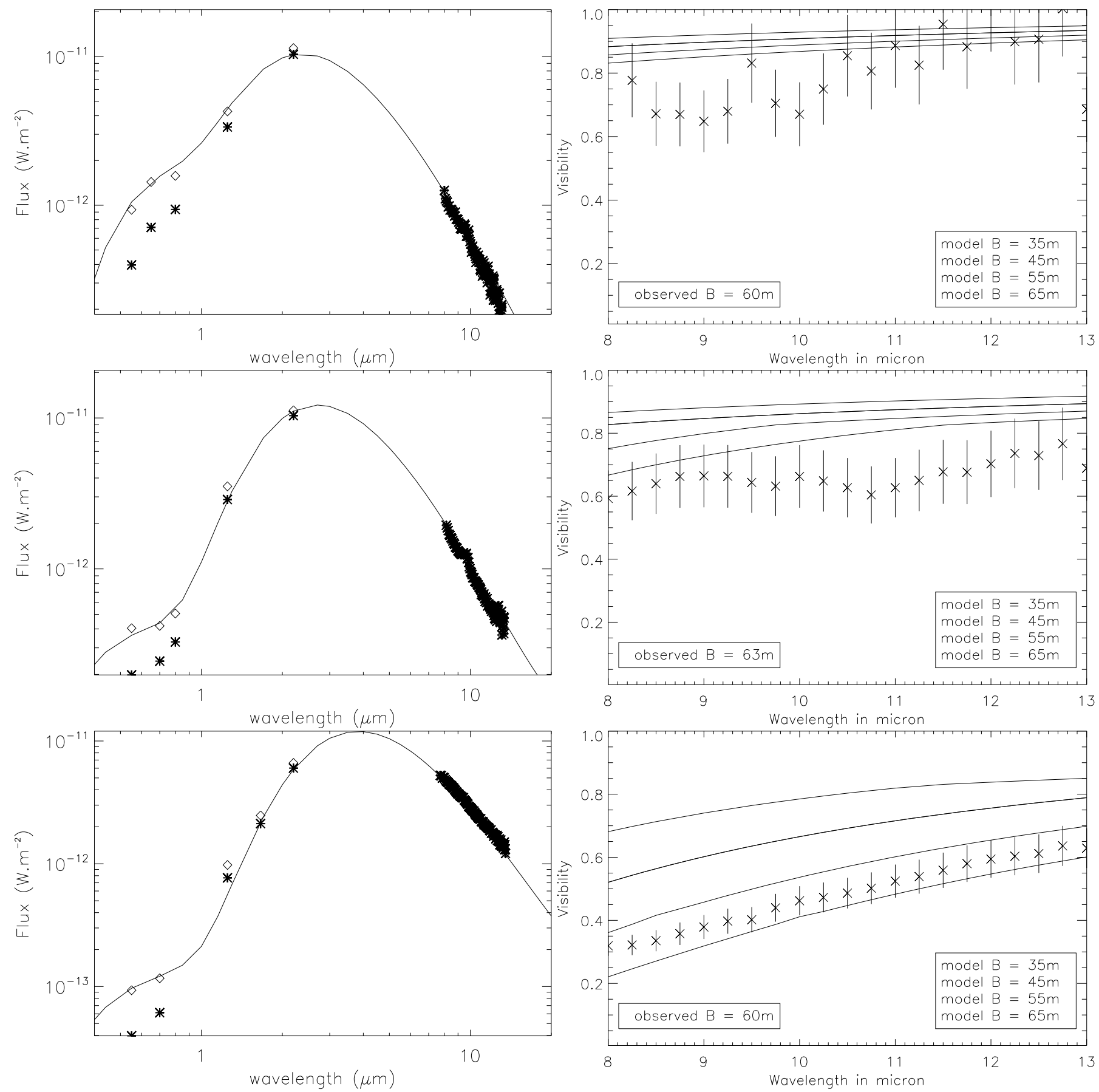

Fig. 6. From top to bottom, SEDs at left and visibilities on right recorded at day 36, 45 and 90 compared to the DUSTY models with parameters described in Table 3. The SEDs are dereddened, assuming $A_{V}=0.9$, changing the observed fluxes (stars) to the corrected ones (squares).

\subsection{Limitations of the model}

The assumptions for a good application of the DUSTY code are not necessarily fulfilled by V1280 Sco. Aspects regarding the symmetry of the source have already been discussed in Sect. 4.3. We concentrate here on the hypothesis that the central source is considered as a point. The study of nova V705 Cas (Evans et al. 2005), using the same code, lies well within the limits imposed by this criterion. Nova V705 Cas was observed 250 days after the outburst when the central source was very hot and compact as seen from a detached, spatially and optically-thin shell. This is not the case for V1280 Sco in the earliest stages, when the dust shell was very close to the central source whose pseudo-photosphere is relatively cold and extended. The pseudophotosphere is most probably surrounded by a diffuse free-free emission zone and at larger distances by a molecular atmosphere close to the dust-forming region leading to a complex Centerto-Limb Variation profile. When the dust shell is optically thin, it is transparent to emission originating in the internal regions that are not point-like and can be resolved by the interferometer. These regions carry their own budget of correlated flux that is far from being taken into account in our models.

In addition, there is a more fundamental limitation to the use of the DUSTY code for distance determination. We consider 

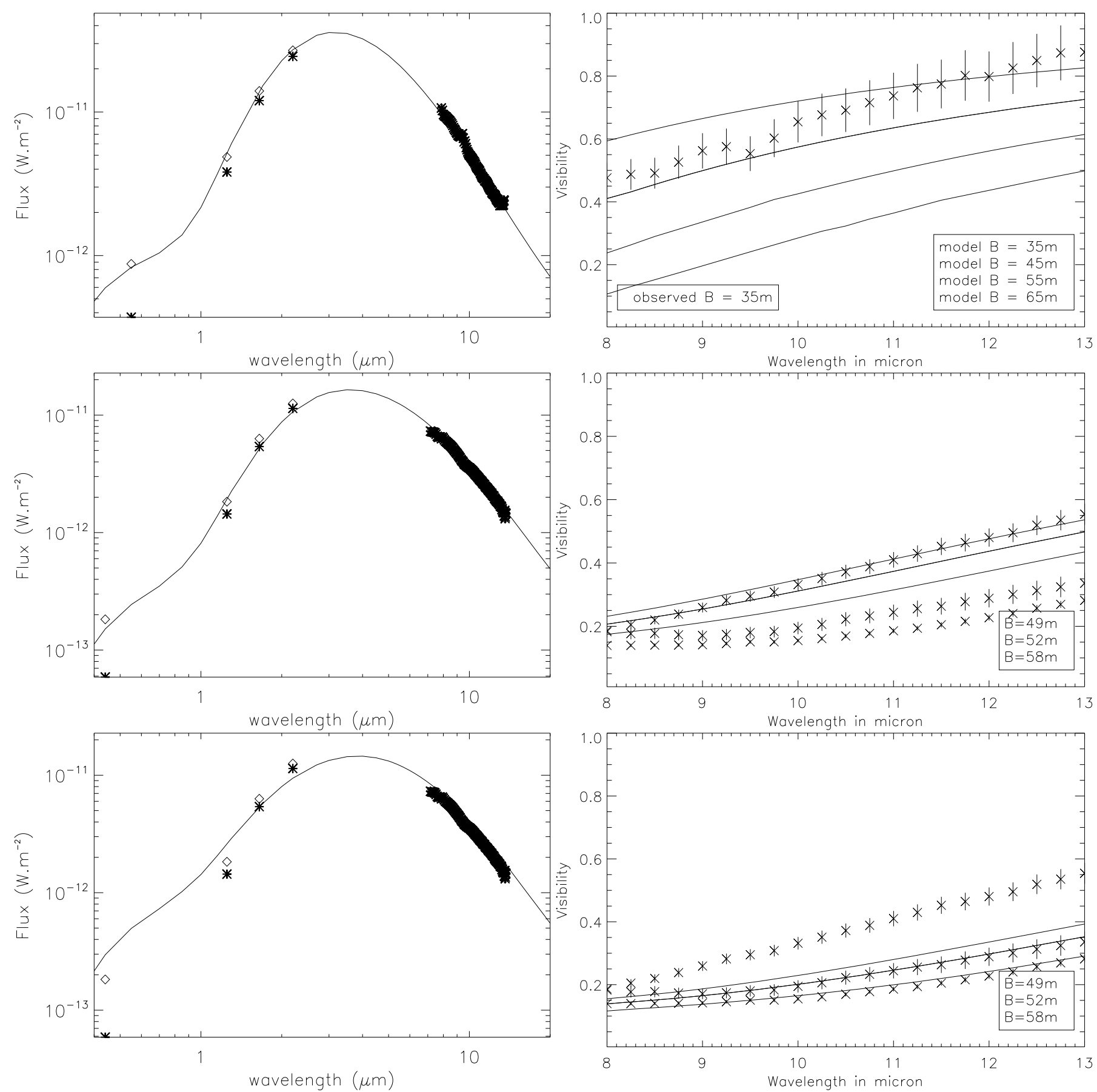

Fig. 7. Same as in Fig. 6 for days 110 and 145. At these dates the presence of a second shell is suspected, and indeed no synthetic visibility curves are found to agree within the full $N$ band spectral range with the high SNR data. The last model is an alternative using a different power law dependence for the density distribution i.e. $r^{-1.1}$ and not $r^{-2}$ as for the other models. For further details see the text.

modeling a dust shell in rapid expansion. The central source is supposed to increase its temperature rapidly, at constant bolometric luminosity. As discussed above, the radius of the shell is not defined in an absolute manner, but by setting a temperature for the central source. Intuitively, we can imagine that as the shell expands, its temperature will steadily decrease; this is not however not observed as can be seen in Fig. 3. This is the wellknown "isothermal" behavior described in Evans et al. (2005, see references there-in) and it directly biases the shell parameters presented in this work. The radius of the dust shell of V705 Cas is found to remain unchanged within the error bars between their epoch $1(t=253 \mathrm{~d})$ and epoch $2(t=320 \mathrm{~d})$ observations, while a $25 \%$ increase is expected by the natural expansion of the shell. This is due to the fact that the shell radius in our DUSTY models is set by the shell temperature and luminosity of the source and does not physically account for the dust facing the hot source (in particular any screening from the gas between the source and the dust). This internal inconsistency prevents us from hoping for a more reliable distance estimate using the model as long as the inner radius is not self-consistently determined in the model accounting for the source characteristics, the shell velocity and an accurate density law. We therefore consider the distance 
Table 3. Model parameters for V1280 Sco for the different epochs using a single dust shell. The parameters (and in particular the luminosity) are scaled for a distance of $1.6 \mathrm{kpc}$. A chronology of dust formation based on an approximate date of dust formation $(t=23 \mathrm{~d})$ estimated from the light curve is also shown. For $t=145 \mathrm{~d}$, two models are proposed which are indicated by designations 145 and $145^{b}$. The second one is more extended and illustrates the degeneracy between models with different shell thicknesses at this stage which is related to the uncertainties in the velocity of the fastest ejecta (see text for discussion).

\begin{tabular}{lcccccc}
\hline \hline & & \multicolumn{5}{c}{ Day after outburst } \\
Days after discovery & 36 & $45 / 46$ & 90 & 110 & 145 & $145^{b}$ \\
Since dust detection & 13 & $22 / 23$ & 67 & 87 & 122 & 122 \\
\hline$T_{*}(K)$ & $8500 \pm 1000$ & $10000 \pm 1000$ & $10000 \pm 1000$ & $10000 \pm 1000$ & $10000 \pm 1000$ & $10000 \pm 1000$ \\
$T_{d}(\mathrm{~K})$ & $1450 \pm 150$ & $1550 \pm 150$ & $1300 \pm 100$ & $1450 \pm 100$ & $1550 \pm 100$ & $1700 \pm 100$ \\
$L\left(L_{\odot}\right)$ & 3500 & 4400 & 6600 & 16000 & 8400 & 8400 \\
$q$ & $2.1 \pm 0.4$ & $2.1 \pm 0.4$ & $2.9 \pm 0.3$ & $2.9 \pm 0.3$ & $3.0 \pm 0.2$ & $3.0 \pm 0.2$ \\
$Y=1+\Delta R / R$ & $1.3 \pm 0.3$ & $1.5 \pm 0.3$ & $2.8 \pm 0.4$ & $3.0 \pm 0.5$ & $5.0_{-1}^{+5}$ & $17^{+3}$ \\
$r_{\text {in }}(\mathrm{mas})$ & 3.4 & 5 & 6. & 6.4 & 3.8 & $2.2^{-6}$ \\
$r_{\text {in }}(\mathrm{AU})$ & 5.4 & 8 & 9.6 & 10.2 & 6.1 & 3.5 \\
$r_{\text {out }}(\mathrm{mas})$ & 4.2 & 6.8 & 16.7 & 19.3 & 29.1 & 37 \\
$\tau_{V}$ & $3 . \pm 0.3$ & $4.5 \pm 0.3$ & $6.5 \pm 0.3$ & $5.4 \pm 0.3$ & $6.2 \pm 1$. & $5.5 \pm 0.4$ \\
$\tau_{10 \mu \mathrm{m}}$ & 2.3 & 4.1 & 2.8 & 2.3 & 2.1 & 1.5 \\
$\tau_{100 \mu \mathrm{m}}$ & $4.43 \times 10^{-2}$ & $6.42 \times 10^{-2}$ & $4.05 \times 10^{-2}$ & $3.27 \times 10^{-2}$ & $2.47 \times 10^{-2}$ & $1.9 \times 10^{-2}$ \\
$M_{\text {dust }}\left(M_{\odot}\right)$ & $4 \times 10^{-8}$ & $1.4 \times 10^{-7}$ & $2.2 \times 10^{-7}$ & $2.2 \times 10^{-7}$ & $1.1 \times 10^{-7}$ & $1 \times 10^{-7}$ \\
\hline
\end{tabular}

estimates based on the uniform disk models to be the most accurate, used during the optically thick regime.

\section{Discussion}

\subsection{Dust shell expansion rate and distance estimation}

This is the first time that the dust shell of a classical nova has been spatially resolved starting from the onset of the shell formation up to the point at which it becomes close to becoming optically thin. This is a new and promising technique for providing dust-shell expansion rates, and thus distances, for interesting novae that are usually much fainter and almost featureless during the time that the dust shell is optically thick. The present data set provides a direct estimate of the distance to V1280 Sco, a target for which a direct application of the MMRD relations is rendered difficult by the dust formation event that appears to have occurred before the light curve dropped below the defined thresholds $t_{2}$ and $t_{3}$.

However, the applicability of such observations must be discussed. First the sphericity of the source is not established from our observations. The second point is that while the VLTI monitored the expansion of the dust shell, the velocities used to infer the distance were measured from visible line measurements. It is not definite that these velocity measurements trace the material that formed dust considering the observational evidence that the filling factor of the ejecta can be far from unity (Ederoclite et al. 2006). Gehrz (1988) provided arguments in favor of the use of the determination of expansion parallaxes in the infrared. The dust forms preferentially in the densest regions of the ejecta, and may follow a different (slower) velocity law than the low-density wind itself. The consistency of the P Cygni absorption positions detected in many lines in the optical and near-IR implies however that the velocity of the dust-forming shell is probably well represented by this value, and the error bar quoted in this paper is a conservative estimate. For the velocity of $575 \mathrm{~km} \mathrm{~s}^{-1}$ reported in Das et al. (2008), the distance scales to $\sim 1.9 \mathrm{kpc}$. This upper estimate must probably be favored in our interpretation. Thirdly, uniform disks are a good approximation for describing the intensity distribution as long as the shell remains optically thick, but simple models are no longer suitable when the shell grows and the optical thickness in the $N$ band becomes low. The data recorded when the shell was optically thick are more robust estimators of the distance despite their lower accuracy.

A distance estimation based on the dust-expansion parallax has some advantages compared to that using the expansion of the fireball in the near-IR as reported in Lane et al. (2007b,a). The near-IR method follows the fireball from its very first moments to the point where the the free-free emission is no longer optically thick. What remains at this stage is a compact, optically thick, shrinking source and an extended free-free emitting halo, rapidly over resolved by the interferometric technique. The time of reaction must be short before observations can begin after being alerted of the early stages of the outburst. In contrast in the mid-IR, the dust shell dominates the flux for a long time, and the contribution of the hot core, even though significant, has less influence on the result. Nevertheless, the near-IR free-free expansion parallax technique has the advantage of being applicable to all novae and not only the ones that form dust.

Our estimate for the time of ejection is subject to some uncertainty, but it appears probable that the material from which the dust is made was launched after the beginning of the outburst, close to the time of maximum light. This does not imply that material was not ejected earlier to this - onset of the eruption is evidenced by P-Cygni profiles observed almost as early as $t_{0}$ which lasted at least for 15 days thereafter: there was indeed a fireball expansion in the early stages. Why did dust not form in these early ejected layers? The temperature of the pseudophotosphere of the firewall is a key parameter, since it probably became colder near and after the peak of visible light. The massloss rate was also probably not constant and might have peaked close to the optical maximum: the dust-formation rate might directly reflect this, although it is questionable whether the second event occurring at $t=110 \mathrm{~d}$ also reflected a sudden increase of the ejected mass.

\subsection{Multiple dust formation events}

Modeling using simple spherical shell models does not clearly establish whether the formation of a second shell occurs from $t=110 \mathrm{~d}$, but the light curve support this hypothesis. Rawlings \& Evans (2002) proposed that the stability and hence the ultimate fate of the grains is primarily determined by the degree to which they are annealed by the nova's ultraviolet radiation field. Shore \& Gehrz (2004) developed this idea arguing that 
the agglomeration of atoms by dust nuclei proceeds kinetically through induced dipole reactions in a partially-ionized medium. Ionization of a cluster increases once the ejecta become transparent in the ultraviolet, which triggers runaway grain growth. The extent to which this mechanism intervenes in the formation of multiple "shells" (i.e. large variations in dust formation rate with time) is unclear. In the case of V1280 Sco, signs of increase of the optical depth due to dust appear very early in the light curve, implying that, once nucleation has occurred, the physical conditions for grain growth (density and thus mass-loss rate and wind speed, temperature of the central source, and perhaps level of soft UV radiations) were optimum. The second shell must also have formed close to the central star. Therefore, its appearance can be considered as independent of the evolution of the first shell. Did the material in between the outbursting source and the dust-forming region dilute below a level in which soft UV radiation emerged again, triggering a new dust nucleation? A more likely hypothesis is an increase in the mass-loss rate (for unknown reasons) or perhaps a change to more favorable chemical conditions (Pontefract \& Rawlings 2004) in conjunction with the hardening of the central source flux. If a 10-15 mas new shell, due to a sudden increase in mass-loss rate, is observed at $t=145 \mathrm{~d}$ then this material was generated about 35-40 days before the observations (assuming still a velocity of $500 \mathrm{~km} \mathrm{~s}^{-1}$ ) i.e. around $t=110 \mathrm{~d}$ when the second light maximum occurred. We can then hypothesize that this second maximum is the consequence of not only the decrease in the expanding dust optical depth but is also related to an increase in the luminosity and mass-loss rate of the central source. We note in Table 3 that the luminosity required to account for the observed data at $t=110 \mathrm{~d}$ is larger than at other epochs.

\section{Conclusions}

High spatial-resolution observations of the very first moments of a nova outburst promise to provide invaluable information about the physical processes operating in the vicinity of the central remnant. The VLTI observations reported here, the first of their kind, show that spectro-interferometric observations can localize accurately the dust-forming regions allowing a "virtual" in-situ study of the dust-forming event.

These observations present interesting information on the dust shell formed around the classical nova V1280 Sco. They still lack however the minimum $u v$ coverage needed to check the sphericity of the source. An opportunity existed to complement the near-IR photometry and spectroscopic data obtained by Das et al. (2008) of this bright source with VLTI/AMBER observations in the same spectral region. The source was sufficiently bright during the first 30 days to be studied with a spectral resolution of 1500 in $H$ and $K$, providing potentially direct constraints on the spatial extent of the line-forming regions and their kinematics (such as in Chesneau et al. 2007). Our short-term involvement in this field and inadequate manpower prevented our team from undertaking these potentially exciting observations - but scope exists for monitoring one or two bright novae per year with this facility. This field, and studies of a similar kind, should benefit from the development of 2nd generation instrumentation of the VLTI. The mid-IR instrument MATISSE (Lopez et al. 2006) is foreseen to recombine the light from 4 telescopes, from $L$ to $N$ band simultaneously, providing in one exposure six visibility measurements and three closure phases per band, that should provide in a single observation a detailed view of a dust-forming shell.

Acknowledgements. The VLTI staff is warmly thanked for their intensive efforts in efficiently operating the facility. By constantly communicating the latest observational results to our team, they helped us to plan and understand the observations. This research has made use of the AFOEV database, operated at CDS, France. We also acknowledge with thanks the variable star observations from the AAVSO International Database contributed by observers worldwide. The blog of the KANATA 1.5-m telescope at Higashi-Hiroshima Observatory, Japan has also provided valuable information for the planning and preliminary understanding of our observations. The research work at Physical Research Laboratory is funded by the Department of Space, Government of India.

\section{References}

Banerjee, D. P. K., Janardhan, P., \& Ashok, N. M. 2001, A\&A, 380, L13 Barry, R. K., Danchi, W. C., Traub, W. A. et al. 2008, [arXiv:0801:4165] Blöcker, T., Balega, Y., Hofmann, K.-H., \& Weigelt, G. 2001, A\&A, 369, 142 Bode, M. F., Harman, D. J., O’Brien, T. J., et al. 2007, ApJ, 665, L63 Cassatella, A., Lamers, H. J. G. L. M., Rossi, C., et al. 2004, A\&A, 420, 571 Chesneau, O., Nardetto, N., Millour, F., et al. 2007, A\&A, 464, 119

Das, R. K., Ashok, N. M., \& Banerjee, D. P. K. 2007, Central Bureau Electronic Telegrams, 866, 1

Das, R. K., Ashok, N. M., \& Banerjee, D. P. K. 2007, Central Bureau Electronic Telegrams, 864, 1

Das, R. K., Banerjee, D. P. K., Ashok, N. M., \& Chesneau, O., 2008, MNRAS, accepted

della Valle, M., \& Livio, M. 1995, ApJ, 452, 704

Deroo, P., van Winckel, H., Verhoelst, T., et al. 2007, A\&A, 467, 1093

Ederoclite, A., Mason, E., Della Valle, M.,et al. 2006, A\&A, 459, 875

Evans, A., Geballe, T. R., Rawlings, J. M. C., \& Scott, A. D. 1996, MNRAS, 282, 1049

Evans, A., Geballe, T. R., Rawlings, J. M. C., et al. 1997, MNRAS, 292, 192

Evans, A., Tyne, V. H., Smith, O., et al. 2005, MNRAS, 360, 1483

Gehrz, R. D. 1988, ARA\&A, 26, 377

Gehrz, R. D., Greenhouse, M. A., Hayward, T. L. et al. 1995, ApJ, 448, L119

Gehrz, R. D., Truran, J. W., Williams, R. E., \& Starrfield, S. 1998, PASP, 110, 3

Harrison, T. E., Johnson, J. J., Mason, P. A., \& Stringfellow, G. S. 1998, ASP Conf. Ser., 137, 489

Ivezic, Z., \& Elitzur, M. 1997, MNRAS, 287, 799

Ivezic, Z., Nenkova, M., \& Elitzur, M. 1999, [arXiv: astro-ph/9910475]

Lane, B. F., Sokoloski, J. L., Barry, R. K. et al. 2007a, ApJ, 658, 520

Lane, B. F., Retter, A., Eisner, J. A., et al. 2007b, ApJ, 669, 1150

Lopez, B., Wolf, S., Lagarde, S., et al. 2006, Proc. SPIE, 6268

Marshall, D. J., Robin, A. C., Reylé, C., Schultheis, M., \& Picaud, S. 2006, A\&A, 453, 635

Mathis, J. S., Rumpl, W., \& Nordsieck, K. H. 1977, ApJ, 217, 425

Millour, F., Petrov, R., Malbet, F., Tatulli, E., \& Duvert, G., 2007, [arXiv:0705.1636]

Monnier, J. D., Barry, R. K., Traub, W. A., Lane, B. F., \& Akeson, R. L. 2006, ApJ, 647, L127

Munari, U., Valisa, P., Dalla Via, G., \& Dallaporta, S. 2007a, Central Bureau Electronic Telegrams, 852, 1

Munari, U., Siviero, A., Henden, A., et al. 2007b, Central Bureau Electronic Telegrams, 1099, 1

Naito, H., \& Narusawa, S. 2007, IAU Circ., 8803, 2

O'Brien, T. J., Bode, M. F., Porcas, R. W., et al. 2006, Nature, 442, 279

Petrov, R. G., Malbet, F., Weigelt, G., et al. 2007, A\&A, 464, 1

Pontefract, M., \& Rawlings, J. M. C. 2004, MNRAS, 347, 1294

Quirrenbach, A., Elias, N. M., Mozurkewich, D., et al. 1993, AJ, 106, 1118

Rawlings, J. M. C., \& Evans, A. 2002, Classical Nova Explosions, 637, 270

Rudy, R. J., Dimpfl, W. L., Lynch, D. K., et al. 2003, ApJ, 596, 1229

Rudy, R. J., Lynch, D. K., Russell, R. W., et al. 2007, IAU Circ., 8809, 1

Sarkar, G., \& Sahai, R. 2006, ApJ, 644, 1171

Schöller, M., Argomedo, J., Bauvir, B., Blanco-Lopez, L., \& Bonnet, H. 2006, Proc. SPIE, 6268, 19

Shore, S. N., \& Gehrz, R. D. 2004, A\&A, 417, 695

Tatulli, E., Millour, F., Chelli, A., et al. 2007, A\&A, 464, 29

Wade, R. A., Harlow, J. J. B., \& Ciardullo, R. 2000, PASP, 112, 614

Yamaoka, H., Fujii, M., \& Naito, H. 2007, IAU Circ., 8807, 2

Yamaoka, H., Nakamura, Y., Nakano, S., Sakurai, Y., \& Kadota, K. 2007, IAU Circ., 8803, 1 